\title{
Spermatozoïdes, environnement et prévention
}

\author{
M. AUROUX, E. DULIOUST, D. FENEUX \\ Biologie de la Reproduction et du Développement, CHU Bicêtre, Le Kremlin-Bicêtre
}

\section{RESUME}

Au cours des 50 dernières années, les facteurs de risque conduisant à l'infertilité ont nettement augmenté, surtout ceux liés à l'environnement. La spermatogénèse et les spermatozoïdes sont susceptibles d'être affectés par des agents physiques et chimiques, toxiques ou médicamenteux, mais aussi par des facteurs naturels comme le stress ou l'âge paternel. Ce dernier facteur et les précédents peuvent évidemment être intriqués. Leur ensemble aboutit à une baisse de la fertilité masculine et il est ainsi possible de définir des populations à risque.

Ces altérations de la spermatogénèse peuvent en outre entraîner des anomalies chez la progéniture tels qu'avortements précoces, chute du poids de naissance, syndromes autosomiques dominants aboutissant à des malformations ou à des troubles fonctionnels, en particulier comportementaux, troubles de la spermatogénèse analogues à ceux présentés par le père, leucémies etc.

De telles données devraient conduire à un renforcement de la prévention et au dépistage de l'hypofertilité avant la trentaine.

Mots clés : spermatozoïde, environnement, fertilité, progéniture, prévention.

\section{INTRODUCTION}

Dans un article récent, les danois [16] tirent la sonnette d'alarme : de 1940 à 1990 , la numération des spermatozoïdes serait passée de 113 millions à 66 millions $/ \mathrm{ml}$. A moins que la qualité des gamètes ne soit également touchée, cette chute du nombre n'a rien de dramatique et 66 millions de bons spermatozoïdes suffisent largement à assurer une descendance. En revanche, la question qui se pose de manière aiguë, compte tenu de la toxicité progressivement croissante de notre environnement, est celle de l'origine de la chute et des éventuelles altérations gamétiques qui pourraient l'accompagner. On sait que, chez l'homme, différents facteurs peuvent modifier la spermatogénèse et entraîner une hypofertilité plus ou moins accentuée, voire une stérilité. Certains, iatrogènes ou toxiques, sont effectivement liés à l'environnement du sujet, d'autres correspondent à des altérations naturelles tenant par exemple au vieillissement, et les effets de ces deux types de facteurs sont évidemment intriqués. Ainsi, relativement à ces situations, il existe des populations à risques chez lesquelles une évaluation précoce des fonctions reproductrices permettrait d'éviter, plus tard, que les individus concernés soient confrontés à une dysfertilité sans ressource thérapeutique. Dans cette perspective, nous allons donner quelques exemples de facteurs capables de perturber la spermatogénèse et d'aboutir à des altérations morphologiques ou fonctionnelles des spermatozoïdes.

Enfin, tout ce qui concerne les spermatozoïdes intéressant non seulement l'individu mais aussi sa descendance, nous envisagerons les risques d'éventuelles mutations dues à ces facteurs. 


\section{ALTERATION DES SPERMATOZOIDES}

\section{Influence de l'environnement}

\section{a] Les facteurs iatrogènes}

Un certain nombre d'agents chimiques et physiques utilisés en thérapeutique sont capables de perturber la spermatogénèse [62]. On a par exemple montré qu'un sulfamide, la salicylazo-sulfapyridine, utilisée dans le traitement de la recto-colite hémorragique, pouvait entraîner des anomalies de la tête des spermatozoïdes réversibles, semble-t-il, après arrêt du traitement [54] et que les nitrofuranes (antibiotiques de synthèse) entraînaient térato-et asthénozoospermie chez l'homme et l'animal [3]. Par ailleurs certains antifongiques, comme le kétoconazole sont, à doses élevées et prolongées, responsables d'un blocage de la sécrétion de testostérone aboutissant, chez l'homme et l'animal, à une azoospermie réversible [1]. Le cas du diethylstibloestrol est particulier puisque ses effets nocifs se sont manifestés via la mère mais on connaît aujourd'hui l'effet délétère qu'a pu avoir cette hormone sur la spermatogénèse de l'enfant $[28,29]$. Les radiations [41] et les antimitotiques utilisés en cancérologie sont plus radicaux et entrấnent, selon les agents employés, une altération telle de la spermatogénèse qu'ils conduisent très souvent à la stérilité. La réversibilité des perturbations dépend des modalités du traitement et des individus. C'est ainsi que, 5 ans après l'administration de chimiothérapies tels que le MOPP (méchloréthamine, vincristine, procarbazine et prednizone) ou le MVPP (méchloréthamine, vinblastine, procarbazine et prednisone), $80 \%$ des hommes demeurent azoospermiques [19].

La cryopréservation du sperme avant la thérapie, quand elle est possible, est actuellement le seul recours vis-à-vis de ces effets secondaires. Toutefois, et bien que ses conséquences soient infiniment plus discrètes, la cryoconservation peut aussi être nocive : elle est par exemple capable d'altérer la mobilité [48] et l'ultrastructure [24] des spermatozoïdes. En outre l'ADN n'est pas épargné, tant en ce qui concerne sa structure [47] que sa méthylation [7] et l'on suppose que l'augmentation de la production de radicaux libres dus au froid est susceptible de l'altérer $[25,40]$.

Pour certains auteurs $[36,63]$ les ultrasons seraient également toxiques, altérant la numération, la morphologie et la mobilité des spermatozoïdes. Les U.S. ont ainsi été proposés comme moyen anticonceptionnel. Ces troubles, qu'on a d'ailleurs décrits comme inconstants [53], sont en général réversibles à l'arrêt du traitement bien que la persistance d'une tératozoospermie ait été signalée [63].

Chez l'animal, les travaux expérimentaux réalisés avec les substances antimitotiques $[57,58,60,61]$, les rayonnements [18] et les U.S. [31, 36] confirment cette susceptibilité de la spermatogénèse. Ils montrent par ailleurs que les tranquillisants du type des benzodiazépines peuvent aussi entraîner des malformations des spermatozoïdes [59], ce qui devient inquiétant si l'on se réfêre, en France, au niveau de consommation de ce genre de substances.

\section{b) Les toxiques}

Chez l'homme, certains pesticides comme le dibromochloropropane [62] ou le dibromure d'éthylène [46] entraînent des altérations de la numération, de la morphologie et de la mobilité des spermatozoïdes. On sait par ailleurs que le plomb, à fortes doses, peut être à l'origine de troubles importants de la spermatogénèse [60]. Le problème reste ouvert pour les doses faibles, telles que celles qui concernent chaque citadin du fait des gaz d'échappement des voitures [52]. D'autres métaux lourds tels que le cadmium, le mercure, le manganèse et le cuivre sont également toxiques. Le cadmium, utilisé dans l'industrie et dans la fabrication des engrais phosphorés, inhibe l'activité de la choline acétyltransférase et diminue, in vitro, la mobilité des spermatozoïdes chez l'homme et le rat [23]. Le méthyl-mercure entraîne une réduction de la fertilité des souris mâle [39] 
mais, jusqu'à présent, aucune conséquence n'a été notée chez l'homme [38]. En revanche l'exposition aux poussières de manganèse entraînerait une réduction de la fertilité [38]. Enfin le cuivre diminue, in vitro, la mobilité des spermatozoïdes humains $[14,32]$.

Le sulfure de carbone, employé dans la fabrication des fibres artificielles, entraînerait chez l'homme une oligo-asthénotératozoospermie qui pourrait être réversible, quoique certains sujets puissent présenter une aggravation des troubles avec le temps. Cette substance agirait sur les cellules de Leydig et sur l'axe hypothalamo-hypophysaire [35, 37].

Chez l'animal, des solvants industriels comme l'éthylène glycol, le mono ou le diméthyl éther provoquent, lorsqu'ils sont inhalés par le mâle chez la souris, le rat, le chien et le lapin, une atrophie de l'épithélium germinal, des anomalies de la tête des spermatozoïdes et une chute de la fertilité [30].

Dans le cadre des toxiques alimentaires, le rôle délétère de l'alcool est démontré chez l'homme lorsque l'alcoolisme s'accompagne de cirrhose : certaines recherches font alors état d'atrophie testiculaire et d'une chute de la numération et de la mobilité des spermatozoïdes [51]. D'autres études, cependant, qui ne considèrent pas le cas des grands alcooliques, ne notent pas de différences entre les buveurs et les non buveurs [51]. Chez le rat, l'alcool entraîne une atrophie testiculaire et une diminution du nombre des cellules germinales [56] ; chez la souris, on note une altération de la numération, de la mobilité et de la morphologie des spermatozoïdes, ainsi qu'une diminution de leur aptitude à féconder l'ovocyte [6]. On a pu enfin, toujours chez la souris, mettre en évidence une hypoploïdie dans les spermatogonies de souris alcoolisées, l'intensité de l'effet étant proportionnel à la dose d'alcool totale administrée [4].

En ce qui concerne le tabac, les résultats sont assez flous et parfois contradictoires, les facteurs en cause (âge, constitution du fumeur etc.) étant nombreux et intriqués. Toutefois, pour un âge égal ou inférieur à 35 ans, la proportion de fumeurs serait significativement plus élevée chez les hommes inféconds que chez les sujets féconds (57\% vs 43\%) [51].

Enfin, certains auteurs se sont intéressés aux drogues psycho-actives, comme le cannabis. Cette substance, administrée à des souris mâles, entraîne une chute du poids des testicules, une altération de la spermatogénèse comportant des anomalies chromosomiques au stade des spermatocytes I et une diminution de la fertilité [20].

\section{c) Les radiations}

En ce qui concerne les radiations, l'effet sur la spermatogénèse des faibles doses reçues à proximité des sources industrielles n'est pas encore précisément connu, mais il scmble qu'il existe et soit responsable de mutations, comme nous le verrons plus loin [27]. Les expositions chroniques ont un effet subtil mais net. Ainsi les radiologistes présentent une diminution significative du taux standardisé de fécondité et, à l'intérieur de cette anomalie, il existe un effet dose [34]. Les employés des usines d'uranium présenteraient pour leur part des oligo-asthénotératozoospermies de degré variable [44].

\section{d) La chaleur}

On a prouvé, chez l'homme et l'animal, que la chaleur perturbait la spermatogénèse et pouvait diminuer la fertilité masculine [42]. Une fièvre élevée et prolongée, un varicocèle important peuvent ainsi être responsables de difficultés plus ou moins graves. Les hommes exposés à des températures élevées du fait de leur profession constituent évidemment une population à risques et l'on a observé que les ouvriers servant les fours à céramiques présentaient une asthénozoospermie expliquant peut-être une augmentation significative des couples sans enfants dans cette population [26]. Notons cependant que l'effet de la chaleur parait réversible. 


\section{Influence des facteurs naturels}

\section{a) L'âge}

La plus grande étude réalisée jusqu'ici [50] montre que, chez l'homme, la numération ne présente pas de variation notable entre 21 et 50 ans. En revanche la morphologie et la mobilité varient selon une parabole dont le sommet correspond à la trentaine. Parallèlement, la fertilité masculine baisse régulièrement à partir de la quarantaine [5] et il n'est pas exclu que cette dégradation due au vieillissement soit plus marquée chez certains sujets à fertilité limite. Actuellement, le recul progressif de l'âge de la paternité peut donc amplifier les difficultés chez des hommes dont la fertilité est naturellement ou accidentellement compromise. Une situation est à cet égard très démonstrative : celle de l'IVG regrettée. Un couple vient consulter parce qu'il n'arrive pas à obtenir l'enfant qu'il désire. Dans les années antérieures une IVG a été pratiquée. Les résultats du spermogramme expliquent les difficultés actuelles. Informé, le couple regrette l'IVG. La question que l'on peut alors se poser, lorsque les couples sont stables, est de savoir si l'examen de sperme ne devrait pas être systématiquement proposé avant l'interruption volontaire de grossesse : en effet, la découverte d'une hypofertilité pourrait faire reconsidérer l'intervention. De plus, cette déficience étant plus facile à corriger chez un sujet jeune, la précocité du diagnostic serait bénéfique. On peut d'ailleurs tenir le même raisonnement avant le début d'une contraception féminine afin de vérifier si, compte tenu des résultats du partenaire, elle est justifiée.

\section{b) Le stress}

Le stress pourrait, selon certains [53], avoir une influence nocive sur la fertilité. C'est en augmentant le taux des enképhalines, lesquelles inhibent la dopamine dont la chute entraîne à son tour une baisse de production du LH-RH, que le stress diminuerait transitoirement la fertilité [22].

\section{RETENTISSEMENT DE L'ALTERATION DES GAMETES MALES SUR LA PROGENITURE}

La diminution de la fertilité masculine due aux facteurs que nous venons d'envisager est une chose mais, en cas de fécondation, le retentissement de l'altération des gamètes sur le développement de la progéniture est un second problème qui amplifie considérablement l'importance du premier.

\section{Influence de l'environnement \\ a) Les facteurs iatrogènes}

Dans ce domaine, les résultats, jusqu'ici, sont surtout expérimentaux. Des travaux déjà anciens ont montré que la thalidomide, la méthadone ou la morphine pouvaient, administrées au mâle, entraîner des perturbations chez la progéniture : augmentation des taux de mortalité foetale et néonatale, poids de naissance abaissé et troubles de l'émotivité [Rev. Gén. 8].

D'autres recherches, fondées sur l'effet de certains antimitotiques ont montré que ces substances, administrées au mâle, pouvaient entraîner des troubles chez sa progéniture. C'est ainsi que la procarbazine entraîne un taux significativement augmenté de pertes embryonnaires post-implantation [57] ; que le cyclophosphamide (Endoxan*) entraîne des malformations quand les croisements sont effectués pendant ou à la fin du traitement [55], mais aussi des troubles essentiellement fonctionnels lorsque les croisements ont été effectués à distance de ce traitement. Il s'agit soit de troubles du comportement chez des rats prépubères [2] soit, comme nous l'avons nous-mêmes mis en évidence, d'une diminution des capacités d'apprentissage chez les adultes [12] accompagnée d'une altération de certains des supports biochimiques de la mémoire [11]. Ces troubles, qui peuvent se transmettre d'une génération à l'autre (nous les avons observés jusqu'à la 3è génération), présentent les caractères de syndromes dominants, ce qui pose évidemment des problèmes sérieux. 
Il semble par ailleurs que la cryoconservation puisse être à l'origine de mutations puisque nous venons nous-mêmes de montrer que les embryons de souris dérivant, cette fois, d'ovocytes congelés, présentaient un taux d'échange des chromatides soeurs anormalement élevé [15]. La question reste de savoir si les spermatozoïdes peuvent être concernés par ce phénomène.

\section{b) Les facteurs toxiques}

Chez l'homme, une enquête anglaise relativement récente [21] traite de l'emploi des solvants industriels dont nous avons déjà parlé. Elle montre que le poids de naissance des enfants nés de pères travaillant en présence de ces substances est significativement plus faible que celui des enfants d'une population témoin.

Expérimentalement, le cannabis peut, administré chez la souris mâle, entraîner une augmentation des morts pré et post natales chez sa progéniture [20]. La situation se complique, en outre, du fait de l'héritabilité possible de certaines des anomalies constatées : en effet, les altérations de la spermatogénèse paternelle peuvent être retrouvées chez la progéniture, ce qui fait redouter la survenue de mutations [20]. Le problème est identique avec le plomb et les benzodiazépines qui provoquent des troubles de la spermatogénèse communs au père et à la progéniture $[60,61,59]$. Dans la même perspective, mais avec une symptomatologie différente, l'alcool parait pouvoir être mutagène car, chez le rat et la souris, l'administration de ce toxique à des mâles entraîne des troubles du comportement chez leurs petits [1].

\section{c) Les radiations}

D'après une enquête anglaise [27], la probabilité d'apparition de leucémies ou de lymphomes serait significativement plus élevée chez les enfants nés de pères professionnellement exposés aux radiations des centrales nucléaires que chez les enfants nés de pères non exposés. Les expériences faites chez l'animal ont par ailleurs montré qu'aux altérations constatées de la spermatogénèse pouvait correspondre une augmentation significative des résorptions embryonnaires $[33,49]$, de diverses malformations et de tumeurs surtout pulmonaires [43].

\section{d) La chaleur}

Chez le bélier, les données expérimentales montrent que si les spermatozoïdes épididymaires, soumis à une légère augmentation de température, sont capables de féconder, le taux de survie des embryons formés est significativement diminué [42]. La question des avortements précoces chez les femmes de sujets exposés à une température extérieure élevée devrait donc être envisagée.

\section{Influence des facteurs naturels \\ a) L'âge}

On sait que, chez l'homme, le vieillissement paternel peut accroître, chez les enfants, le risque d'apparition de syndromes autosomiques dominants (achondroplasie, syndromes d'Apert, de Marfan, maladie de Recklinghausen etc...) ou même récessifs pour certains auteurs (hémophilie A, myopathie de Duchenne) [Rev. Gén. : 10].

Expérimentalement, nous avons mis en évidence que le vieillissement paternel peut en outre déterminer, chez la progéniture du rat, des troubles essentiellement fonctionnels tels qu'une baisse des capacités d'apprentissage des petits devenus adultes [9]. Nous avons en outre décelé un phénomène analogue dans l'espèce humaine où, pour un âge maternel donné, la réussite d'hommes de 18 ans à des tests psychométriques décroit à mesure que s'élève l'âge du père à la naissance [13]. De façon remarquable, la réussite à ces tests est également pénalisée par un très jeune âge paternel. La courbe des résultats présente ainsi, globalement, la forme d'une parabole dont le sommet correspond à un âge paternel d'environ 30 ans, rappelant en cela l'évolution des caractères qualitatifs des spermatozoïdes [50]. 


\section{CONCLUSION}

Ces quelques exemples montrent que certains facteurs, naturels ou artificiels et appartenant à l'environnement, peuvent altérer la spermatogénèse humaine. Les études expérimentales confirment la réalité d'une telle toxicité.

La première conséquence de cette altération est une chute de la fertilité. On peut ainsi définir des populations à risques, selon les facteurs en cause. De ce point de vue, et compte tenu de l'évolution de la spermatogénèse avec l'âge, le dépistage de l'hypofertilité aurait avantage, tant du point de vue de l'individu que de celui de l'économie de la santé, à être effectué avant 30 ans. Lorsque les couples sont stables, il pourrait être systématiquement recommandé avant les IVG et le début d'une contraception féminine.

La seconde conséquence correspond, au-delà de l'hypofertilité, au retentissement de ces différents facteurs sur le développement d'un éventuel conceptus.

Le problème que nous avons abordé présente ainsi deux aspects, le second n'étant pas le moins important. La gravité des données acquises et des questions posées nous paraissent rendrc nécessaire leur prise en compte médico-sociale.

\section{BIBLIOGRAPHIE}

1. ABEL E.L. : Paternal Behavioral Mutagenesis. Neuro Toxicology, 1989, $10: 335-346$.

2. ADAMS P.M., FABRICANT J.D., LEGATOR M.S. : Cyclophosphamide induced spermatogenetic effects detected in the $\mathrm{F} 1$ generation by behavioral testing. Science 1981, $211: 80-83$.

3. ALI BH. Pharmacology and toxicity of furarolidone in man and animals : some recent research. Gén. Pharmacol. 1989, 20 : 557-563.

4. ALVAREZ M.R. : Numerical chromosome variation in mouse spermatogonia resulting from alcohol consumption. J. Heredity, 1983, $74: 58-61$.

5. ANDERSON B.A. : Male age and infertility. Result from Ireland prior to 1911. Pop. Index 1975, $41: 561-567$.
6. ANDERSON R.A., WILLIS B.R., OSWALD C., ZANEVELD LJ. : Ethanol-induced male infertility : impairment of spermatozoa. J. Pharmacol. Exp. Therapeut., 1983, $225: 479-486$.

7. ASCHWOOD-SMITH M.J. : The cryopreservation of human embryos. Hum. Reprod. 1986, 1 : 319-322.

8. AUROUX M. : La physiotératogénèse du comportement. Un nouvel aspect de la pathologie du développement prénatal. J. Gyn. Obst. Biol. Repr. 1981, $10: 633-640$

9. AUROUX M. : Decrease of learning capacity in offspring with increasing paternal age in the rat. Teratology, 1983, 27 : 141-148.

10. AUROUX M. : Age du père et développement. Contracept. Fertil. Sex. 1993, $21: 382-385$.

11. AUROUX M., DULIOUST E., NAWAR N.N.Y., YACOUB S.G., KEMPF E.H., EBEL A.B. : Cyclophosphamide in the male Rat : cerebral biochemical changes in progeny. Biomed. Pharmacother. $1990,44: 519-523$.

12. AUROUX M., DULIOUST E., SELVA J., RINCE P. : Cyclophosphamide in the Fo male Rat : physical and behavioral changes in three successive adult generations. In : "Male mediated $\mathrm{F} 1$ abnormalities". Mutat. Res. 1990, $229: 189-200$.

13. AUROUX M., MAYAUX M.J., GUIHARD-MOSCATO M.L., FROMANTIN M., BARTHE J., SCHWARTZ D. : Paternal age and mental functions of progeny in man. Hum. Reprod. 1989, 4 : 794-797.

14. BATTERSBY S., CHANDLER J.A., MORTON M.S. : Toxicity and uptake of heavy metals by human spermatozoa. Fertil. Steril. 1982, $37: 230$ 235.

15. BOUQUET M., SELVA J., AUROUX M. : Cryopreservation of Mouse Ovocytes : Mutagenic Effects in the Embryo ? Biol. Reprod., 1993, 49 : 764-769.

16. CARLSEN E., GIWERCMAN A., KEIDING N., SKAKKEBAEK NE. : Evidence for decreasing quality of semen during past 50 years. Brit. Med. J. 1992, Sept. 12, 305:609-613.

17. DE COSTER R. : Endocrinological effect of single daily ketoconazole administration in male beagle dogs. Acta Endocrinol. 1989, 53 : 203-205.

18. COX B.D., LYON M.F. : The induction by X-Rays of chromosome aberrations in germ cells of male guinea-pigs, golden hamsters and rabbits. I. Dose response in post-meiotic stages. Mutat. Res., 1975, 29 : 93-109.

19. CZYGLIK F. : Autoconservation de sperme avant thérapeutique stérilisante : indications et résultats. In : L'insémination artificielle, CECOS ed. Masson, PARIS, 1991, 53-61. 
20. DALTERIO S., DAIR F., BARTKE A. and MAYFIELD D. : Cannabinoids in male mice : Effects on fertility and spermatogenesis. Science 1982, 216 : 315-316.

21. DANIELL W.E., VAUGHAN T.L. : Paternal employment in solvent related occupations and adverse pregnancy outcomes. British J. Indust. Med. 1988, 45 : 193-197.

22. DUNBAR R. : Stress as a good contraceptive. New Scientist. 1985, $17: 16-18$.

23. DWIVEDI C. : Cadmium induced sterility possible involvement of the cholinergic system. Arch. Environ. Contam. Toxicol. 1983, 12 : 151-156.

24. ESCALIER D., BISSON J.P. : Quantitative ultrastructural modifications in human spermatozoa after freezing. In : David G., Price W. eds. Human artificial insemination and semen preservation. New York : Plenum Press, 1980, 107 122.

25. FERRADINI C., PUCHEAULT J. : Biologie de l'action des rayonnements ionisants. Paris, Masson 1983.

26. FIGA-TALAMANCA I., DELL'ORCO V., PUPI A., DONDERO F., GANDINI L., LENZI A., LOMBARDO F., SCAVALLI P., MANCINI G. : Fertility and semen quality of workers exposed to high temperatures in the ceramics industry. Reprod. Toxicol. $1992,6: 517-523$.

27. GARDNER M.J., SNEE P.M., HALL A.J., POWELL A.C., DOWNES S., TERRELL J.D. Results of case-control study of leukemia and lymphona among young people near Sellafied nuclear plant in West Cumbria. Brit. Med. J. 1990, 300 : 423-429.

28. GILL W.B., SCHUMACHER G.F.B., BIBBO M. : Pathological semen and anatomical abnormalities of the genital tract in human male subjects exposed to diethylstilbestrol in utero. J. Urol. 1977, $117,477-480$.

29. GILL W.B., SCHUMACHER G.F.B., BIBBO M., STRAUS F.H., SHOENBERG H.W. : Association of diethylstilbestrol exposure in utero with cryptorchidism, testicular hypoplasia and semen abnormalities. J. Urol. 1979, 122 : 36-39.

30. HARDIN B.D. : Reproductive Toxicity of the glycol ethers. Toxicology 1983, $27: 91-102$.

31. HILL C.R., CLARK P.R., GROWE M.R., HAMMICK J.E. : Biophysical effects of cavitation in a 1 $\mathrm{MHz}$ ultrasonic beam. Ultrasonics for industry 1969. Conference papers : $26-30$.

32. HOLLAND M., WHITE I.G. : Heavy metals and spermatozoa. 1. Inhibition of the mobility and metabolism of spermatozoa by metals related to copper. Fertil. Steril. 1980, $34: 483-489$.
33. JEGOU B., VELEZ de la CALLE J.F., BAUCHE F. : Protective effect of medroxyprogesterone acetate plus testosterone against radiation-induced damage to the reproductive function of male rats and their offspring. Proc. Natl. Acad. Sci. USA, $1991,88: 8710-8714$.

34. KITABATAKE T., WATWABE T., SATO T. : Sterility in japanese radiologicol technicians. Tahoku J. Exp. med., 1974, 42 : 209-212.

35. LANCRANJAN I. : Alterations of spermatic liquid in patients chronically poisoned by carbon disulphide. Med. Lav. 1972, 63 : 29-33.

36. LANCRANJAN I., MAIGANESCU M., RAFAILA E. : Gonadic function in workmen with long term exposure to microwaves. Health Phys. 1975, 29 : 381-383.

37. LANCRANJAN I., POSPESCU H.I, KLEPSCH $J$. : Changes of the gonadic function in chronic carbon disulphide poisoning. Med. Lav. 1969, 60 : 566-571.

38. LAUWERYS R., ROELS H., GENET P., TOUSSAIN G., BOUCKAERT A., DE COOMAN S. : Fertility of male workers exposed to mercury vapor or to manganese dust - a questionnaire study. Am. J. Ind. Med. 1985, $7: 171-176$.

39. LEE I.P., DIXON R.L. : Effects of mercury on mouse spermatogenesis studied by cell separation and serial mating. Toxicol. Appl. Pharmacol. 1973, 25 : 464.

40. LEIBOVITZ B., SIEGEL B.V. : Aspect of free radical reactions in biological systems. Aging J. Gerontol $1980,35: 45-56$.

41. MFISTRICH M.L., SAMUELS R.C. : Reduction in sperm levels after testicular irradiation of the mouse : a comparison with man. Radiat. Res., 1985,102 : 138-147.

42. MIEUSSET R. : Effets de la température sur les fonctions testiculaires et épididymaires. Thèse 3è cycle Biologie de la Reproduction, Université de Paris XI, CHU Bicêtre, 1992.

43. NOMURA T. : Parental exposure to X-rays and chemicals induces heritable tumours and anomalies in mice. Nature, 1982, $296: 575-577$.

44. POPESCU H.I., LANCRANJAN I. : Spermatogenesis alteration during protected irradiation in man. Health Phys., 1975, 28 : 567-573.

45. RAO B., DAVID G. : Improved recovery of postthaw motility and vitality of human spermatozoa cryopreserved in the presence of dithiothreitol. Cryobiology 1984, 21, 536-541.

46. RATCLIFFE J.M., SCHRADER S.M., STEENLAND K., CLAPP D.E., TURNER T., HORNUNG R.W. : Semen quality in papaya workers with long term exposure to ethylene dibromide. Brit. J. Indust. Med., 1987, $44: 317-326$. 
47. ROYERE D., HAMAMAH S., NICOLLE J.C., BARTHELEMY C., POINDRON J., ROUSSIE M., LANSAC J. : Effets de la congélation sur la stabilité de la chromatine des spermatozoïdes humains : étude cytophotométrique. Fertil. Contracep. Sexual 1987, 15 : 718-721.

48. SERRES C., JOUANNET P., CZYGLIK F., DAVID G. : Effects of freezing on spermatozoa motility. In : David G., Price W. eds. Human artificial insemination and semen preservation. New York : Plenum Press, 1980, 147-160.

49. SCHRODER J.H., HUG O. : Dominante letal mutationen in der nachkommenschaft bestrahlter mänulicher mäuse. Mutat. Res. 1971, 11 : 215-245.

50. SCHWARTZ D., MAYAUX M.J., SPIRA A., MOSCATO ML., JOUANNET P., CZYGLIK F., DAVID G. : Semen characteristics as a function of age in 833 fertile men. Fertil. Steril. 1983, $39: 530-535$.

51. SPIRA A., DUCOT B., JOUANNET P., SOUMAH A., FENEUX D., ALBERT M. : Consommation de tabac, d'alcool et fertilité masculine. In : Facteurs de la stérilité humaine, A. SPIRA et P. JOUANNET Eds. INSERM 1981, Vol. $103: 363-372$.

52. STANWELL-SMITH R., THOMPSON S.G., HAINES A.P., WARD RJ., CASHMORE G., STEDRONSKA J., HENDRY WF. : A comparative study of zinc, copper, cadnium and lead levels in fertile and infertile men. Fertil. Steril. 1983, $40:$ 670-677.

53. STEENO O.P. PANGKAHILA A. : Occupational influences on male fertility and sexuality. Androl., 1983, $16: 5-22$.

54. TOTH A. : Reversible toxic effect of salicylazosulfapyridine on semen quality. Fertil. Steril. 1979, 31 : 538-540.

55. TRASLER J.M., HALES B.F., ROBAIRE B. : Paternal cyclophosphamide treatment of rats causes foetal loss and malformations without affecting male fertility. Nature 1985, 316:144-146.

56. VAN THIEL D.H., GAVALER J.S., COBB C.F., SHERINS RJ., LESTER R. : Alcohol induced testicular atrophy in the adult male rat. Endocrinology, $1979,105: 888-895$.

57. VELEZ de la CALLE JF., JEGOU B. : Protection by steroid contraceptives against procarbazineinduced sterility and genotoxicity in male rats. Cancer Res., 1990, 50 : 1308-15.

58. VELEZ de la CALLE JF., SOUFIR JC., CHODORGE F., BOISSEAU C., KERCRET H., JEGOU B. : Reproductive effects of anti-cancer drug procarbazine in male rats at differents ages. J. Reprod. Fertil., 1988, 84 : 51-61.

59. WAR R.N. and DAS R.K. : Induction of sperm head abnormalities in mice by three tranquillizers. Cytobios $1983,36: 45-51$.
60. WYBOREK A.J. and BRUCE W.R. : The induction of sperm-shape abnormalities in mice and humans. In CHEMICAL MUTAGENS, Vol. 5, A. Hollaender and F.J. de Serres eds. Plenum Pub. Corp. NEWYORK 1978, 257-285.

61. WYBOREK A.J., GORDON L.A., BURKHART J.G., FRANCIS MW., KAPP RW., LETZ G., MALLING HV., TOPHAM JC., WHORTON MD. : An evaluation of the mouse sperm morphology test and other sperm tests in non human mammals. Mutat. Res. 1983, $115: 1-72$.

62. WYBOREK A.J., GORDON L.A., BURKHART J.G., FRANCIS MW., KAPP RW. Jr, LETZ G., MALLING HV., TOPHAM JC., WHORTON MD. : An evaluation of human sperm as indicators of chemically induced alteration of spermatogenic function. Mutat. Res. 1983, $115: 73-148$.

63. YU-HU L., ZHI-QIANG L., YAO-QUN W., HUAN BING L., BING-REN F. : Long term effect on human spermatogenesis after microwaves treatment of testes. J. Bioelectricity, 1988, $7: 97-102$.

\section{ABSTRACT}

\section{Spermatozoon, environment and prevention}

\section{Auroux, E. Dulioust, D. Feneux \\ Biologie de la Reproduction et du développement, CHU Bicêtre, Le Kremlin-Bicêtre}

Over the last fivty years, the risk factors for infertility have increased substantially, particularly those due to the environment. Spermatogenesis and spermatozoa can be affected by physical (ionizing radiation microwaves, heat, cryopreservation) or chemical agents (antimitotics drugs, antibiotics, tranquillizers, insecticides, pesticides, industrial solvants, some heavy metals, alcohol, cannabis etc.). Some natural factors, as stress or paternal age (ageing or very youthful age relatively to about thirty) also seems to affect spermatogenesis and, particularly, the age can be joined with the previous ones. On the whole, these factors are able to decrease the male fertility through some changes about the concentration, the motility or the morphology of spermatozoa and so it is 
possible to describe populations subject to the risk.

Moreover, these spermatogenetic changes can lead abnormalities in progeny. For instance, some antimitotic drugs as cyclophosphamide, when administrated to the male rat, lead malformations or functional anomalies as behavioral troubles. The industrial solvents lead a decrease of the birth weight and the cannabis leads an increase of the ante-or post-natal death. Moreover, the change of the paternal spermatogenesis caused by cannabis can be found again in the male progeny. The problem is similar with the lead, the benzodiazepines and the alcohol.

Concerning the physical factors, some authors have shown that the children born from radiation exposed fathers presented an increase of the probability of leukemia. In animal, the postimplantation loss is increased when the father is irradiated or subjected to heat before mating.

Finally, the paternal ageing is responsible for new dominant autosomic mutations. Moreover, in animal and man, paternal ageing and, in man, very youthful age, also seems responsible for a gradual lowering in the level of progeny cerebral functions.

On the whole, these data should lead to an preventive attitude which would be more effective before about thirty years of age than after this period.

Key-words : spermatozoon, environment, fertility, progeny, prevention. 